$\mathrm{E}$ EVALUAR
2017, Vol. 17, No 2.

ISSN 1667-4545

Recuperado de https://revistas.unc.edu.ar/index.php/revaluar

Laboratorio de Evaluación Psicológica y Educativa

Facultad de Psicología - Universidad Nacional de Córdoba

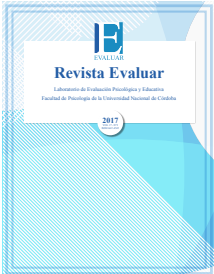

\title{
Evaluación de un Instrumento de Apoyo Institucional en Casos de Maltrato Infantil
}

\author{
Analysis of an Instrument for Assessing Institutional Support \\ in Cases of Child Maltreatment
}

\author{
Valeria Arredondo * ${ }^{1}$, Carolina Saavedra ${ }^{1}$, Cristóbal Guerra ${ }^{1}$ \\ 1 - Centro de Estudios en Infancia, Adolescencia y Familia, ONG Paicabi, Chile.
}

Introducción

Método

Resultados

Discusión

Referencias

Recibido: 22/08/2017 Revisado: 27/09/2017 Aceptado: 11/10/2017

\section{Resumen}

En Chile no existen suficientes instrumentos que evalúen de forma confiable y válida los factores involucrados en el maltrato infantil. En particular hay carencia de instrumentos que se centren en el rol que juegan las instituciones gubernamentales y no gubernamentales en la protección y apoyo a niños maltratados. Por esta razón el objetivo del estudio fue evaluar las propiedades psicométricas de un instrumento para evaluar el apoyo social institucional en casos de maltrato infantil grave (I-APSI). Se consideraron los datos de 544 niños, niñas y adolescentes atendidos en centros especializados en Chile. Se aplicaron el I-APSI y dos instrumentos que evalúan constructos relacionados. Los resultados apoyan la validez factorial del instrumento, su validez convergente y su confiabilidad. Pese a lo alentador de estos resultados, se debe considerar que el estudio es preliminar y se debe seguir profundizando en las propiedades psicométricas del instrumento.

Palabras clave: maltrato infantil, apoyo social, protección, confiabilidad, validez

\begin{abstract}
There are not enough reliable and valid instruments to evaluate the factors involved in child maltreatment in Chile. In particular, there is a lack of instruments that focus on the role of governmental and non-governmental institutions in protecting and supporting abused children. For this reason, the objective of the study was to evaluate the psychometric properties of an instrument that assesses institutional social support in cases of severe child maltreatment (I-APSI). Data from 544 children and adolescents that were receiving treatment in specialized centers in Chile was contemplated. I-APSI and two instruments assessing related constructs were applied. The results support the factorial validity of the instrument, its convergent validity and its reliability. These results should be considered as preliminary, and the psychometric properties of the instrument should be further investigated in the future.
\end{abstract}

Key words: children maltreatment, social support, protection, reliability, validity

*Correspondencia a: valeria.arredondo@paicabi.cl

Cómo citar este artículo: Arredondo, V., Saavedra, C., \& Guerra, C. (2017). Evaluación de un instrumento de apoyo institucional en casos de maltrato infantil. Revista Evaluar, 17(2), 165-176. Recuperado de https://revistas.unc.edu.ar/index.php/revaluar 


\section{Introducción}

El maltrato infantil, dentro del cual se incluyen la negligencia, el maltrato psicológico, el maltrato físico y el abuso sexual, es una de las formas más graves de vulneración de derechos contra menores de 18 años (UNICEF, 1989). Se considera que sus consecuencias son de alto riesgo para el desarrollo y bienestar de los niños y niñas, dado que quienes lo experimentan evidencian dificultades en casi todas las dimensiones evolutivas (Morelato, 2011), entre las que se encuentran la presencia de depresión, baja autoestima, agresividad, desesperanza, escasas habilidades de afrontamiento, dificultades en el control de impulsos y regulación emocional y peor desempeño académico en comparación con los niños que no han sido víctimas de malos tratos en su vida. Debido justamente a las serias consecuencias emocionales, sociales, cognitivas y comportamentales que genera en sus víctimas, el maltrato infantil es un hecho rechazado socialmente en la mayoría de los países en forma cada vez más clara y sostenida (Barudy, 1998), aspecto que se instala en la agenda internacional a partir de la Convención Internacional de los Derechos del Niño (UNICEF, 1989), que en su articulado determina el rechazo al trato duro o violento hacia los niños, comprometiendo a los estados a generar las políticas públicas necesarias para su implementación. En coherencia con esta visibilidad social del maltrato infantil, en 1990 el Estado de Chile ratifica dicha Convención $\mathrm{y}$, mediante este acto, se compromete a velar por el respeto a los derechos de los menores de 18 años en el país. Este compromiso abarca a todas las instituciones del Estado, quienes se ven obligadas a considerar dentro de sus lineamientos una posición coherente con la promoción de derechos de la infancia, la protección de sus intereses y necesidades, y la reparación integral tras la vulneración de sus derechos fundamentales.
En este escenario, si bien es posible considerar que el Estado de Chile ha avanzado en este cambio de paradigma hacia la protección especializada de la infancia tras la Convención Internacional de los Derechos del Niño, a través de la generación de políticas públicas focalizadas, la creación de centros de atención especializados para cubrir los procesos reparatorios tras la identificación de la vulneración de derechos y avances en la legislación en infancia, adolescencia y familia (Guerra \& Arredondo, 2017), también es posible indicar que las tasas de maltrato infantil en Chile siguen siendo elevadas pese a estos esfuerzos. En este sentido, estudios chilenos estiman que el $75.1 \%$ de los niños y niñas del país habrían sido maltratados al menos alguna vez en su vida (Ministerio del Interior, 2013). Por su parte, el Cuarto Estudio de Maltrato Infantil, desarrollado por UNICEF en Chile en el año 2012, basado en la aplicación de cuestionarios autoadministrados a niños y niñas en establecimientos educacionales, al comparar los resultados de este estudio con los obtenidos en 1994, 2000 y 2006; señala que el $71 \%$ de los niños y niñas recibe algún tipo de violencia de parte de su madre y/o padre, un 51.5 $\%$ sufre algún tipo de violencia física y el $25.9 \%$ de los niños y niñas sufre violencia física grave. Cabe destacar que, si bien los datos ilustran una disminución de la violencia general hacia los niños y niñas entre los años 1994 y 2012, la violencia física grave se ha mantenido sin modificaciones (UNICEF, 2012). Por su parte, un reciente estudio realizado con adolescentes en Chile indica que el $89 \%$ de un total de 706 participantes informa que ha sufrido algún tipo de victimización o maltrato a lo largo de su vida (Pinto-Cortez \& Venegas-Sanhueza, 2015). En este estudio se destaca la experiencia de hasta seis acontecimientos distintos de maltrato en el $68.1 \%$ de los casos, en tanto que el $30.3 \%$ declara haber experimentado siete o más eventos de maltrato o violencia. Estos 
datos indican que el maltrato ocurre más en episodios cotidianos repetidos que en eventos aislados, aspecto que resulta fundamental de considerar a la hora de generar un marco comprensivo del fenómeno para su enfrentamiento e intervención.

Ahora bien, Cichetti, Rogosch, Lynch y Holt (1993) sostienen que no todos los niños y niñas son afectados del mismo modo por estas experiencias de maltrato infantil, pues estas dependen de su edad y período de desarrollo, así como de los contextos y formas de maltrato, es decir que cada experiencia está configurada en un entramado de condiciones que se asocian a su impacto específico. En esta línea, si consideramos el maltrato infantil como una experiencia adversa que implica un riesgo de sufrir distintos tipos de consecuencias, Luthar, Cicchetti y Becker, (2000) plantean que pueden existir variaciones en el funcionamiento y desarrollo de los individuos expuestos a este tipo de experiencias, enfatizando que podrían manifestar competencia en algunas áreas y exhibir algunas dificultades en otras en un patrón más bien variable. Lo anteriormente expuesto es un elemento crucial que debe ser considerado por los distintos servicios que brindan atención y asistencia a niños, niñas y adolescentes que han experimentado maltrato, dado que a partir de este tipo de consideraciones se puede diseñar una ayuda atingente a las necesidades y posibilidades de cada niño o niña víctima, considerando sus particularidades. En la temática del maltrato infantil, uno de los aspectos que es importante integrar es el apoyo social, dado que constituye una variable destacada en la protección contra el maltrato infantil y también está asociado a su impacto, en la medida que tendría una función mediadora o moderadora entre la experiencia de maltrato y el desarrollo de sintomatología psicológica (Evans, Steel, \& DiLillo, 2013; Guerra, Ocaranza, \& Weinberger, 2016; Guerra, Pereda, Guilera, \& Abad, 2016; Pereda-Beltran, 2009;
Turner, Shattuck, Finkelhor, \& Hamby, 2015), debido a lo cual su consideración dentro de los procesos de intervención resulta relevante.

El apoyo social puede ser entendido como el proceso interaccional por el cual los recursos en la estructura social (como la comunidad y redes sociales) hacen posible la satisfacción de distintos tipos de necesidades en situaciones cotidianas y de crisis (Gracia \& Musitu, 1993). Dentro de las posibilidades de análisis del apoyo social, se encuentra la consideración de distintas fuentes de origen y mantención, tales como la red de relaciones sociales donde se encuentran las relaciones íntimas, con vecinos, amigos, compañeros de trabajo, entre otros, y las organizaciones y servicios formales e informales, que comprenden las asociaciones cívicas, servicios sociales y de apoyo profesional, entre otras (Gracia \& Musitu, 1993). Otro enfoque de análisis de esta variable es el de la perspectiva relacional vinculada con el niño o niña. Así, Luthar y Goldstein (2004) señalan que los padres de los niños y niñas, así como otros adultos presentes en su contexto de vida, pueden proveer y proveer un valioso soporte frente a las situaciones de adversidad. Por ello, se debe alentar la relación con adultos de confianza, lo cual contribuiría a disminuir las consecuencias de los malos tratos en los niños y adolescentes; este aspecto debe ser considerado dentro de la intervención. Otros han considerado lo opuesto, es decir que más que disminuir las consecuencias de los malos tratos, la relación con adultos de confianza es un factor que contribuye al bienestar por sí solo, lo que le otorga valor en el desarrollo de las necesidades de afiliación, pertenencia, respeto, $\mathrm{y}$ reconocimiento social (Gracia \& Musitu, 1993). En cualquier caso, su consideración como variable relevante dentro de la evaluación e intervención en maltrato infantil queda establecida.

No obstante, una de las dificultades para los centros de atención especializada en atención a 
víctimas de maltrato infantil grave es que no se cuenta con instrumentos confiables y válidos para evaluar los avances de las intervenciones y las condiciones de esta variable necesarias para abordar la problemática en términos reparatorios (Arredondo, Saavedra, \& Guerra, 2017). Desde este punto de vista, se considera pertinente aumentar los esfuerzos por diseñar o adaptar instrumentos que puedan ser utilizados en Chile, de manera fiable y que resulten ser una guía para el diseño de la intervención.

Debido a lo anterior, la ONG Paicabi (2012) diseñó un instrumento para evaluar una dimensión específica del apoyo social institucional, la visibilización del niño y de su familia. Este apoyo es otorgado por organismos sociales ante situaciones de maltrato infantil grave (I-APSI). La relevancia de evaluar el apoyo social institucional está situada en dos aspectos que convergen. El primero, un nivel netamente ético-político vinculado con el seguimiento de los postulados de la Convención de los Derechos del Niño (UNICEF, 1989) que, como ya se ha dicho, obliga a todas las instituciones de los estados adscritos a velar por la protección de los niños y niñas, y ejercer un rol fiscalizador y reparador ante una situación de vulneración de derechos como lo es el maltrato infantil. En este sentido, la red institucional formal comprende una red de recursos para la superación del maltrato como problema social. El segundo, un nivel pragmático asociado con la evidencia empírica que pone en relieve la importancia del apoyo social. En este sentido, se decidió generar un instrumento que evalúe el apoyo de las redes sociales institucionales debido a la consideración de que el apoyo social es una de las variables que más frecuentemente se destacan en la literatura como factor protector ante situaciones y consecuencias del maltrato (Evans et al., 2013; Guerra, Ocaranza, et al., 2016; Guerra, Pereda, et al., 2016; Turner et al., 2015). De hecho, se plan- tea que los soportes extrafamiliares constituyen un factor asociado con la resiliencia en niños y niñas que han sufrido violencia, por lo cual su consideración en el análisis de las victimizaciones infantiles tiene alcances evaluativos e interventivos (Morelato, 2011). Este factor comprende los entornos que brinden sostén desde el contexto comunitario del niño o niña (escolar, familiar, religioso, etc.): actividades extracurriculares, experiencias positivas en la escuela y la posibilidad de tener un entorno estable y/o una comunidad religiosa de referencia. Estos entornos promueven el desarrollo de factores de resiliencia en niños y adolescentes. Es importante tener en cuenta que este factor se combina e interactúa con ciertos aspectos individuales tales como adecuadas habilidades cognitivas y autoestima. En este sentido, es importante señalar que los aspectos individuales asociados con la resiliencia pueden haberse desarrollado justamente gracias a la presencia de alguna forma de protección en los contextos de cuidado (familias sustitutas o un buen ambiente escolar, por ejemplo), especialmente durante la infancia temprana, aspecto que ha sido relevado por distintos autores (Egeland, Carlson, \& Sroufe, 1993; Valentine \& Feinahuer, 1993).

Considerando todo lo anterior, el I-APSI fue elaborado por la ONG Paicabi a través de la colaboración de distintos profesionales expertos (trabajadores sociales y psicólogos) que atienden a niños, niñas y adolescentes que han sufrido maltrato infantil grave. Su finalidad es constituir una herramienta útil para la valoración de los factores de apoyo social institucional vinculados con la visibilización del niño, niña o adolescente y así contribuir a determinar los objetivos de la intervención. La versión inicial fue creada en el año 2003 por un comité de expertos de la misma institución y contó con un total de 12 ítems. Desde ese año se han realizado 3 pilotajes que culminaron en el año 2012 con la versión final, que cuenta 
con un total de 10 ítems. El instrumento es heteroaplicado por los profesionales a cargo de la atención directa de los niños y niñas participantes de los centros especializados. Para poder aplicarlo de forma adecuada es necesario que los profesionales se capaciten en ello basándose en el manual creado para tal efecto (Paicabi, 2012).

Si bien desde el año 2012 a la fecha el I-APSI ha sido aplicado por los profesionales responsables del proceso de atención especializada al interior de la ONG, aún no se han estudiado las propiedades psicométricas de este instrumento. Por esta razón, el objetivo del estudio es evaluar las propiedades psicométricas del I-APSI, considerando los datos tomados en función de niños, niñas y adolescentes chilenos. En particular se evalúa la estructura factorial del instrumento, confiabilidad y validez convergente mediante el análisis de la relación con otras dos medidas que evalúan algunas variaciones del apoyo del entorno social (específicamente el reconocimiento y protección de la red institucional ante situaciones de maltrato). Se espera que el instrumento demuestre una adecuada consistencia interna y una estructura factorial compatible con la teoría y que sus puntuaciones se asocien directamente con las otras medidas de apoyo social.

\section{Método}

Participantes

En este estudio se evaluó el apoyo social institucional recibido por niños, niñas y adolescentes que se encontraban en intervención por maltrato infantil grave en centros especializados. Para ello, se consideraron los datos de las fichas clínicas de 544 niños, niñas y adolescentes atendidos en once centros de intervención especializada en maltrato infantil grave de la ONG Paicabi, de la $\mathrm{V}^{\circ}$ Región en Chile. Únicamente se consideraron los casos de aquellos niños, niñas y adolescentes de los cuales se tenía un completo diagnóstico de los factores involucrados en el presente estudio.

Del total de casos analizados, 339 (62.3\%) eran de género femenino y 205 (37.7\%) eran de género masculino. Las edades de los participantes fluctuaron entre 1 y 17 años $(\mathrm{M}=10.13$; DE = 3.99). Del total de casos, 288 (52.9\%) habían sido víctimas de delitos sexuales, 109 (20\%) habían sido testigos de violencia intrafamiliar, 78 (14.3\%) habían sufrido maltrato físico y 67 (12.3\%) maltrato psicológico.

\section{Instrumentos}

En este estudio se utilizaron 3 instrumentos. El primero de ellos es el instrumento para evaluar el apoyo social institucional en casos de maltrato infantil grave, cuyo análisis psicométrico es el objetivo del estudio. Los otros dos instrumentos han sido incluidos como medidas de constructos asociados, relevantes para el proceso de validez convergente del I-APSI.

\section{Instrumento para evaluar el apoyo social insti-} tucional en casos de maltrato infantil grave (IAPSI). El instrumento debe ser respondido por el equipo profesional tras la evaluación diagnóstica de cada niño, niña o adolescente en base a su protocolo de aplicación (Paicabi, 2012). La versión aplicada en este estudio posee 10 ítems. El formato de respuesta de cada ítem ofrece 3 alternativas, donde: 1 = Baja presencia del indicador, 2 = Presencia moderada del indicador y $3=$ Alta presencia del indicador. Las propiedades psicométricas del instrumento se describen en los siguientes apartados.

Factor contextual del instrumento para evaluar el reconocimiento de situaciones de maltrato infantil I- REC (Paicabi, 2012). Se trata de 
una prueba heteroaplicada de 7 ítems que evalúa en qué medida la red de apoyo institucional que rodea al niño es capaz de reconocer el carácter abusivo de situaciones de maltrato infantil grave (ejemplo de indicador: la red focal identifica los derechos vulnerados del niño, niña o adolescente). El formato de respuesta de cada ítem ofrece 3 alternativas, donde: $1=$ Baja presencia del indicador, 2 = Presencia moderada del indicador y 3 = Alta presencia del indicador. Los puntajes posibles varían entre los 7 y 21 puntos (a mayor puntuación, mayor reconocimiento de situaciones de maltrato infantil). El instrumento posee adecuadas propiedades psicométricas en población chilena (Arredondo, Saavedra, \& Guerra, 2017). En este estudio el instrumento obtuvo una buena confiabilidad ( $\alpha=.88$ ).

Factor contextual del instrumento para evaluar indicadores de protección en el contexto de maltrato infantil grave I-PROT (Paicabi, 2012; Arredondo-Ossandon, Saavedra-Inostroza, \& Guerra-Vio, 2016). Se trata de un instrumento heteroaplicado de 5 ítems que evalúa los recursos protectores del contexto social institucional ( $p$. ej., presencia de redes focales que visibilicen el maltrato). El formato de respuesta de cada ítem ofrece 3 alternativas, donde: 1 = Baja presencia del indicador, 2 = Presencia moderada del indicador y 3 = Alta presencia del indicador. Los puntajes posibles varían entre los 5 y 15 puntos (a mayor puntuación mayor presencia de indicadores proteccionales). En este estudio el instrumento obtuvo una buena confiabilidad $(\alpha=.86)$.

\section{Procedimiento}

Previo a su implementación, el proyecto fue aprobado por un comité de ética institucional compuesto por profesionales especializados en el área y externos al equipo de investigación.
Una vez seleccionados los niños, niñas y adolescentes participantes del estudio, los profesionales respondieron los instrumentos ya especificados. Es necesario señalar que todos los profesionales fueron capacitados sobre cómo responder los instrumentos de este estudio. En todo momento se guardó reserva de los datos de identificación de cada participante.

\section{Análisis de datos}

En primer lugar, los 10 ítems iniciales del I-APSI fueron sometidos a un análisis factorial exploratorio de ejes principales con rotación oblimin. Luego, se evaluó la confiabilidad de los factores resultantes mediante el cálculo del coeficiente alfa de Cronbach y alfa ordinal (Domínguez-Lara, en prensa). En tercer lugar, se evaluó la validez convergente del instrumento mediante el análisis de la correlación entre sus puntuaciones y las de las dimensiones contextuales del I-REC y del I-PROT. Este análisis fue realizado con el coeficiente rho de Spearman. Finalmente, se calcularon puntajes cuartiles para interpretar provisionalmente los resultados de futuras aplicaciones.

\section{Resultados \\ Estructura factorial y confiabilidad}

Previo al análisis factorial se analizó el coeficiente de significación de Kaiser Mayer (KMO $=.80) \mathrm{y}$ el índice del test de esfericidad de Barlett $\left(\chi^{2}=3811,329 ; p<.01\right)$. Ambos análisis sugieren que los ítems están relacionados entre sí, lo que permite su factorización (Vivanco, 1999). El resultado del análisis factorial arrojó 2 factores con autovalores mayores a 1. No obstante, se descartó esta solución ya que el factor 1 estaría conforma- 
Tabla 1

Cargas factoriales en la solución de dos factores y cargas factoriales, relaciones ítem-test y alfa si se elimina el ítem en la solución de un factor.

\begin{tabular}{|c|c|c|c|c|c|}
\hline & \multicolumn{2}{|c|}{ Solución de dos factores } & \multicolumn{3}{|c|}{ Solución de un factor } \\
\hline & $\begin{array}{c}\text { Cargas en } \\
\text { Factor } 1\end{array}$ & $\begin{array}{c}\text { Cargas en } \\
\text { Factor } 2\end{array}$ & $\begin{array}{l}\text { Cargas en } \\
\text { Factor único }\end{array}$ & $\begin{array}{l}\text { Relación } \\
\text { ítems test }\end{array}$ & $\begin{array}{c}\alpha \text { si se } \\
\text { elimina el } \\
\text { ítem }\end{array}$ \\
\hline $\begin{array}{l}\text { Visibilidad del niño(a) o joven en la Red } \\
\text { Educacional }\end{array}$ & .55 & & .74 & .70 & .90 \\
\hline Visibilidad del niño(a) o joven en la Red de Salud & .46 & .39 & .75 & .71 & .90 \\
\hline $\begin{array}{l}\text { Visibilidad del niño(a) o joven en la Red } \\
\text { Institucional }\end{array}$ & .74 & & .79 & .75 & .90 \\
\hline Visibilidad del niño(a) o joven en la Red Judicial & .84 & & .63 & .59 & .91 \\
\hline $\begin{array}{l}\text { Visibilidad del niño(a) o joven en la Red } \\
\text { Comunitaria }\end{array}$ & & .83 & .61 & .60 & .91 \\
\hline Visibilidad de la familia en la Red Educacional & .54 & .31 & .77 & .73 & .90 \\
\hline Visibilidad de la familia en la Red de Salud & .53 & .38 & .81 & .77 & .90 \\
\hline Visibilidad de la familia en la Red Institucional & .70 & & .77 & .72 & .90 \\
\hline Visibilidad de la familia en la Red Judicial & .86 & & .71 & .67 & .91 \\
\hline $\begin{array}{l}\text { Visibilidad de la familia en la } \\
\text { Red Comunitaria }\end{array}$ & & .77 & .64 & .59 & .91 \\
\hline
\end{tabular}

Nota. Sólo se incluyeron cargas factoriales $\geq .3$.

do por 8 ítems y el factor 2 sólo estaría compuesto de 2 ítems, lo que no es recomendable (Kline, 2005). Además, tres de los ítems presentaban cargas factoriales mayores a .3 en más de un factor, lo que sugiere que podría obtenerse una solución de mayor parsimonia (ver Tabla 1).

Efectivamente, al repetir el análisis prefijando la obtención de un factor único, todos los ítems presentan cargas factoriales altas en el factor (sobre .6). El factor único permite explicar el 57.1\% de la variabilidad de los ítems. Además se aprecia que el factor único presenta adecuada confiabilidad $(\alpha$ de Cronbach $=.91 ; \alpha$ ordinal $=.92)$. Todos los ítems se encuentran asociados con la escala total y la eliminación de ninguno de ellos aumentaría la consistencia interna de la escala.
Validez convergente y estadísticos

descriptivos del I-APSI

Como se puede ver en la Tabla 2, la puntuación del I-APSI está directamente relacionada con las puntuaciones de la dimensión contextual del I- REC $(\mathrm{M}=16.48 ; \mathrm{DE}=3.50)$. Como ya se ha señalado, el I-REC evalúa en qué medida la red de apoyo institucional que rodea al niño es capaz de reconocer el carácter abusivo de situaciones de maltrato infantil grave. De esta manera, y de acuerdo con lo esperado, cuanto más visibiliza la red institucional al niño o a su familia mayor tiende a ser el reconocimiento por parte de esta red de las situaciones de maltrato infantil grave.

En la misma línea, la puntuación del I-APSI también se relaciona directamente con las pun- 
tuaciones de la dimensión contextual del I-PROT $(\mathrm{M}=11.92 ; \mathrm{DE}=2.62)$. Esto da cuenta de que a mayor apoyo de las redes sociales institucionales que rodean al niño, niña o adolescente hay mayor disponibilidad de recursos proteccionales en ese contexto, lo que es compatible con las hipótesis planteadas.

\section{Tabla 2}

Relación de las puntuaciones del I-APSI con los constructos asociados y estadísticos descriptivos.

\begin{tabular}{lc}
\hline & I-APSI \\
\hline I-PROT Contextual & $\rho .55^{* *}$ \\
I-REC Contextual & $\rho .51^{* *}$ \\
Media (DE) & $24.06(4.75)$ \\
Asimetría & -.517 \\
Curtosis & -.072 \\
Valor mínimo & 10 \\
Cuartil 1 & 20 \\
Cuartil 2 & 24 \\
Cuartil 3 & 28 \\
Valor Máximo & 30 \\
\hline
\end{tabular}

Nota. Correlaciones rho de Spearman: $* * p<.01$.

La Tabla 2 también muestra datos descriptivos de las puntuaciones del instrumento. Específicamente, la media, desviación típica, valores mínimos, cuartil 1, cuartil 2, cuartil 3, valor máximo, asimetría y curtosis. Estos valores pueden ser usados como referencias para la interpretación de los resultados del instrumento en futuras aplicaciones. A la hora de interpretar las puntuaciones de esta muestra es importante considerar que los valores posibles oscilan entre los 10 y 30 puntos.

\section{Discusión}

El objetivo de este estudio fue evaluar las propiedades psicométricas del I-APSI que, si bien está siendo utilizado en Chile por la ONG Paicabi desde el año 2012, no contaba con respaldo empírico de su confiabilidad ni de su validez. De este modo, la relevancia de la investigación radica en la evidencia otorgada respecto de las garantías psicométricas que posee el instrumento, dado que con él se toman importantes decisiones respecto a las intervenciones de niños, niñas y adolescentes cuyos derechos han sido gravemente vulnerados. Asimismo, la plataforma de intervención en maltrato infantil a nivel nacional carece de este tipo de instrumentos a pesar de que el maltrato infantil es una dimensión de trabajo interventivo definida desde la política pública en infancia presente en todo Chile por normativa técnica nacional (SENAME, 2011), por lo cual se supone que el instrumento resultará útil en este contexto.

En particular, el I-APSI evalúa un constructo escasamente medido por otros instrumentos que evalúan el apoyo social. La mayoría de los instrumentos se centran en la percepción subjetiva de apoyo social del niño, niña o adolescente, sin considerar una medida externa del apoyo institucional (Terol-Cantero et al., 2004). Los instrumentos que teóricamente presentan mayor convergencia con el I-APSI son el I- REC y el I-PROT en sus dimensiones contextuales (Paicabi, 2012) que, aunque evalúan otras dimensiones más específicas del apoyo institucional (reconocimiento de situaciones abusivas y prácticas protectoras respectivamente), no incorporan reactivos referidos a un elemento más elemental como es la visibilización del niño y de su familia.

Se considera que esta omisión en la mayoría de los instrumentos de evaluación del apoyo social genera un importante vacío, especialmente en el contexto del maltrato infantil grave, en el que las condiciones proteccionales de la vida del niño o niña pasan muchas veces por la acción institucional coherente y ajustada a sus necesidades, aspecto fundamental en la intervención especiali- 
zada (Barudy, 1998, 2001). Respecto a este punto, las normativas técnicas a nivel nacional para los programas de atención especializada en maltrato infantil desde el Estado de Chile incorporan esta dimensión tanto en la evaluación como en la acción interventiva (SENAME, 2011); sin embargo, no proponen instrumentos específicos para abordar esta dimensión, lo que crea el vacío que la presente investigación pretendió abordar.

Desde la perspectiva ecológica, se puede entender que en la mantención de las prácticas abusivas en contra de niños, niñas o adolescentes operan una serie de factores individuales, familiares y contextuales para los que las instituciones sociales, especialmente las que provienen del estado, juegan un rol importante (Belsky, 1980; Bronfenbrenner, 1987). De hecho, la Convención de los Derechos del Niño (UNICEF, 1989) apunta precisamente a eso, a que las instituciones del Estado se comprometan por velar por los derechos de los menores de edad, de manera que se disminuya el maltrato en todos sus niveles. Ahora bien, entendiendo que en el abordaje de la problemática del maltrato infantil la primera acción por realizar es tomar una medida protectora que ponga al niño fuera de peligro, estas acciones no son suficientes para la superación de las consecuencias del maltrato. Se ha observado que a medida que transcurre el tiempo suelen manifestarse consecuencias del maltrato a pesar de que los niños ya no están en peligro (Cicchetti \& Rogosch, 1997; Flores, Cicchetti, \& Rogosch, 2005), probablemente porque un factor de protección no es siempre un factor de resiliencia (Cyrulnik, 2003). Por ello, se torna necesario evaluar y consecuentemente intervenir en el apoyo social institucional promoviendo el fortalecimiento de las redes sociales (Morelato, 2011).

Desde este punto de vista, para la ONG Paicabi cobra especial relevancia la planificación de las intervenciones reparatorias a víctimas de maltrato considerando todos los factores involucrados, ya sea maltrato individual, familiar o contextual (Paicabi, 2015). Con esto en mente es que se diseña el I-APSI.

Los resultados de este estudio dan cuenta de que el I-APSI otorga garantías psicométricas para su utilización en Chile. En particular entrega indicios de validez factorial, validez convergente y de confiabilidad (Anastasi \& Urbina, 1998).

La estructura unifactorial sugiere agrupar los 10 ítems en un solo factor. Desde lo teórico se puede decir que esta estructura posee parsimonia, lo que hace recomendable su utilización en la práctica. Además, a nivel empírico esta estructura demuestra alta confiabilidad, lo que indica que las puntuaciones miden de forma consistente el apoyo institucional y que las puntuaciones obtenidas por los sujetos tienden a reflejar verdaderas diferencias en el constructo medido (Oviedo \& Campo-Arias, 2005).

Por otro lado, se aprecia que el I- APSI posee validez convergente ya que su puntuación se asocia en forma directa y significativa con dos medidas de apoyo institucional que se encuentran teóricamente vinculadas (dimensiones contextuales del I-REC e I-PROT). Cabe destacar que la magnitud de estas relaciones es moderada (relaciones del orden del .5) lo que demuestra que, aunque se trata de constructos relacionados, los tres instrumentos miden formas diferentes de apoyo institucional.

Lo anterior pone en relieve la utilidad del I-APSI para medir un aspecto específico y general del apoyo institucional: la visibilización del niño y de su familia, aspecto muy necesario para el posterior reconocimiento y movilización de recursos protectores del entorno. En este estudio se pudo evidenciar cómo, en la medida que la red institucional visibiliza al niño y a su familia, también tiende a demostrar una mayor capacidad para reconocer situaciones de maltrato infantil grave y, 
por ende, para actuar de forma protectora, lo que es altamente deseable en las intervenciones con víctimas de este tipo de vulneraciones contra los niños.

No obstante, por tratarse de un estudio realizado con una muestra relativamente pequeña, es necesario considerar estos resultados como preliminares. Futuros estudios deberán profundizar en los análisis, por ejemplo, mediante un análisis factorial confirmatorio o mediante otras pruebas de validez, como la validez predictiva. En este sentido, sería relevante estudiar el valor predictivo de un alto apoyo de la red social del niño en su proceso de recuperación emocional.

Por último, es necesario destacar que al tratarse de un instrumento heteroaplicado, es necesario que los profesionales que lo utilicen sean entrenados en el formato de su aplicación según las directrices establecidas en el manual (Paicabi, 2012). Se considera que aplicar el instrumento sin el entrenamiento adecuado podría llevar a tomar decisiones erradas respecto del proceso de intervención con niños, niñas y adolescentes cuyos derechos han sido gravemente vulnerados.

\section{Referencias}

Arredondo, V., Saavedra, C., \& Guerra, C. (2017). Análisis psicométrico preliminar de un instrumento para evaluar el reconocimiento de situaciones de maltrato infantil (I-REC) en Chile. Revista de Psicología Clínica con Niños y Adolescentes, 4(2), 111-117. Recuperado de http://www.revistapcna.com/es

Arredondo-Ossandon, V., Saavedra-Inostroza, C., \& Guerra-Vio, C. (2016). Análisis psicométrico preliminar de un instrumento para evaluar indicadores proteccionales en situaciones de maltrato infantil grave (I-PROT). Revista de Trabajo Social, 90, 3-14. Recuperado de http://rts.alerta.cl/index.php/rts/index
Anastasi, A., \& Urbina, S. (1998). Test psicológicos. México: Prentince Hall.

Barudy, J. (1998). El dolor invisible de la infancia. Una lectura ecosistémica del maltrato infantil. Barcelona: Paidós.

Barudy, J. (2001). Maltrato Infantil. Ecología social: Prevención y reparación. Santiago de Chile: Galdoc.

Belsky, J. (1980). Child maltreatment, an ecological integration. American Psychologist, 35(4), 320-335. doi: 10.1037/0003-066X.35.4.320

Bronfenbrenner, U. (1987). La Ecología del Desarrollo Humano. Barcelona: Paidos.

Cicchetti, D., \& Rogosch, F. A. (1997). The role of self-organization in the promotion of resilience in maltreated children. Development and Psychopathology, 9, 797-815. doi: 10.1017/s0954579497001442

Cicchetti, D., Rogosch, F. A., Lynch, M., \& Holt, K. D. (1993). Resilience in maltreated children: Processes leading to adaptive outcome. Development and Psychopathology, 5, 629-647. doi: 10.1017/ s0954579400006209

Cyrulnik, B. (2003). Los patitos feos. La resiliencia: Una infancia infeliz no determina la vida. Madrid: Gedisa.

Dominguez-Lara, S. (en prensa). Fiabilidad y alfa ordinal. Actas Urológicas Españolas.

Egeland, B., Carlson, E., \& Sroufe, L. A. (1993). Resilience as process. Development and Psychopathology, 5(4), 517-528. doi: 10.1017/S0954579400006131

Evans, S. E., Steel, A. L., \& DiLillo, D. (2013). Child maltreatment severity and adult trauma symptoms: Does perceived social support play a buffering role? Child Abuse \& Neglect, 37, 934-943. doi: 10.1016/j. chiabu.2013.03.005

Flores, E., Cicchetti, D., \& Rogosch, F. A. (2005). Predictors of resilience in maltreated and nonmaltreated latino children. Developmental Psychology, 41(2), 338-351. doi: 10.1037/0012-1649.41.2.338

Gracia, E., \& Musitu, G. (1993). El maltrato infantil. Un análisis ecológico de los factores de riesgo. Madrid: Centro de Publicaciones de Ministerio de Asuntos Sociales. 
Guerra, C., \& Arredondo, V. (2017). Investigación sobre psicoterapia en abuso sexual infantil: ¿Una tarea pendiente en Chile? Summa Psicológica 14(1), 1-11. Recuperado de https://summapsicologica.cl/index. php/summa

Guerra, C., Ocaranza, C., \& Weinberger, K. (2016). Searching for social support moderate the relationship between polyvictimization and externalizing symptoms: A brief report. Journal of Interpersonal Violence, (Advance online publication) doi: 10.1177/0886260516642293

Guerra, C., Pereda, N., Guilera, G., \& Abad, J. (2016). Internalizing symptoms and polyvictimization in a clinical sample of adolescents: The roles of social support and non-productive coping strategies. Child Abuse \& Neglect, 54, 57-65. doi: 10.1016/j. chiabu.2016.03.004

Kline, R. B. (2005). Principles and practice of structural equation modeling. New York: Guilford.

Luthar, S. S., Cicchetti, D., \& Becker, B. (2000). The construct of resilience: A critical evaluation and guidelines for future work. Child Development, 71(3), 543562. doi: 10.1111/1467-8624.00164

Luthar, S. S., \& Goldstein, A. (2004). Children's exposure to community violence: Implications for understanding risk and resilience. Journal Clinical Child Adolescence Psychology, 33(3), 499-505. doi: 10.1207/s15374424jccp3303_7

Ministerio del Interior (2013). Encuesta Nacional de Victimización por Violencia Intrafamiliar y Delitos Sexuales. Recuperado de https://estudios.sernam.cl/ documentos/?eMjM1MTAxOQ==Encuesta_Nacional_de_Victimizaci\%C3\%B3n_por_Violencia_Intrafamiliar_y_Delitos_Sexuales_2012

Morelato, G. (2011). Maltrato infantil y desarrollo: Hacia una revisión de los factores de resiliencia. Pensamiento Psicológico, 9(17), 83-96. Recuperado de http://revistas.javerianacali.edu.co/index.php/ pensamientopsicologico
Oviedo, H. C., \& Campo-Arias, A. (2005). Aproximación al uso del coeficiente alfa de Cronbach. Revista Colombiana de Psiquiatría, 34, 572-580.

Paicabi (2012). Protocolo de aplicación de instrumento de medición de indicadores de Apoyo Social Institucional. Manuscrito inédito. ONG Paicabi, Viña del Mar, Chile.

Paicabi (2015). Propuesta técnica ampliada programas reparación maltrato infantil grave. Manuscrito inédito. Viña del Mar, Chile: ONG Paicabi.

Pereda-Beltran, N. (2009). Consecuencias psicológicas iniciales del abuso sexual infantil. Papeles del Psicólogo, 30(2), 135-144. Recuperado de http://www. papelesdelpsicologo.es

Pinto-Cortez, C., \& Venegas-Sanhueza, K. (2015). Experiencias de victimización y polivictimización en jóvenes chilenos. Señales, 9(14), 5-25. Recuperado de http://www.sename.cl/revistas-senales

SENAME (2011). Bases técnicas especificas. Programa de protección especializado. Modalidad maltrato infantil grave y abuso sexual. Santiago de Chile: Sename.

Terol-Cantero, M. del C., López-Roig, S., Neipp-López, M. C., Rodríguez-Marín, J., Pastor, M. A., \& MartínAragón, M. (2004). Apoyo social e instrumentos de evaluación: Revisión y clasificación. Anuario de Psicología, 35(1), 23-45.

Turner, H. A., Shattuck, A., Finkelhor, D., \& Hamby, S. (2015). Effects of poly-victimization on adolescent social support, self-concept, and psychological distress. Journal of Interpersonal Violence, 32(5), 755780. doi: $10.1177 / 0886260515586376$

UNICEF (1989). Convención de los Derechos del Niño. Recuperado el 22 de octubre del 2017 de https://www.unicef.es/causas/derechos-ninos/ convencion-derechos-ninos

UNICEF (2012). $4^{\circ}$ Estudio de Maltrato Infantil en Chile. Análisis comparativo 1994-2000-2006-2012. Santiago: UNICEF. Recuperado de http://unicef.cl/web/ wp-content/uploads/2015/07/Maltrato-Infantil.pdf 
Valentine, L., \& Feinauer, L. L. (1993). Resilience factors asociated with female survivors childhood sexual abuse. The American Journal of Family Therapy, 21, 216-224.

Vivanco, M. (1999). Análisis estadístico multivariable: Teoría y práctica. Santiago, Chile: Editorial Universitaria. 